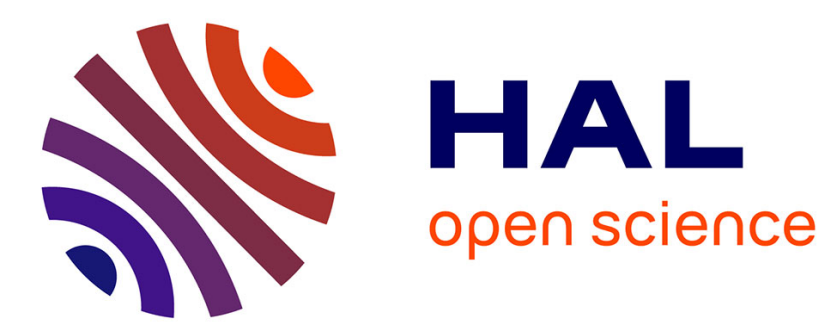

\title{
Live Enrolment for Identity Documents in Europe
} Tarmo Kalvet, Henrik Karlzén, Amund Hunstad, Marek Tiits

\section{To cite this version:}

Tarmo Kalvet, Henrik Karlzén, Amund Hunstad, Marek Tiits. Live Enrolment for Identity Documents in Europe. 17th International Conference on Electronic Government (EGOV), Sep 2018, Krems, Austria. pp.29-39, 10.1007/978-3-319-98690-6_3 . hal-01961517

\section{HAL Id: hal-01961517 \\ https://hal.inria.fr/hal-01961517}

Submitted on 20 Dec 2018

HAL is a multi-disciplinary open access archive for the deposit and dissemination of scientific research documents, whether they are published or not. The documents may come from teaching and research institutions in France or abroad, or from public or private research centers.
L'archive ouverte pluridisciplinaire HAL, est destinée au dépôt et à la diffusion de documents scientifiques de niveau recherche, publiés ou non, émanant des établissements d'enseignement et de recherche français ou étrangers, des laboratoires publics ou privés. 


\title{
Live Enrolment for Identity Documents in Europe
}

\author{
Tarmo Kalvet ${ }^{1}$ Henrik Karlzén ${ }^{2}$ Amund Hunstad ${ }^{3}$ Marek Tiits ${ }^{4}$ \\ ${ }^{1}$ Tallinn University of Technology, Akadeemia tee 3, Tallinn 12618, Estonia \\ ${ }^{2}$ Swedish Defence Research Agency, SE-164 90 Stockholm, Sweden \\ ${ }^{3}$ Swedish Defence Research Agency, SE-164 90 Stockholm, Sweden \\ ${ }^{4}$ Institute of Baltic Studies, Lai 30, Tartu 51005, Estonia \\ tarmo.kalvet@ttu.ee
}

\begin{abstract}
Digital image alterations (morphing) of identity document photos is a major concern and may potentially allow citizens with malicious intent to enrol for identity document(s) later to be used also by another individual. Taking the photo in the application office - live enrolment - can address this issue. However, this is a break with tradition and entails a sizeable overhaul in the public sector, which can be reluctant to change and often lacks the necessary formal methods that ensure a smooth transition. The objective of this paper is to map the main barriers and drivers related to live enrolment based on theoretical research and interviews conducted with high-ranking officers at passport authorities in Estonia, Kosovo, Norway and Sweden. These countries have successfully switched to live enrolment. The main driver for live enrolment has been increased security; for Estonia, user convenience was important and was behind the decision of keeping alternative application processes for the citizens around. The absence of legacy systems makes it easier to implement public sector innovations, such as live enrolment. Behind the successful implementation is proper risk management, covering technological, political and organisational risks. Finally, the research results indicate varying experiences, obstacles, cultural differences and tradeoffs, emphasizing the need to understand barriers and drivers in a contextualised way.
\end{abstract}

Keywords: Morphing, Identity document, Passport, Live enrolment, Social acceptance, Public sector innovation, Drivers and barriers, Risk management

\section{Introduction}

Existing facial recognition algorithms are unable to fully deal with digital image alterations. This means that malicious citizens are able to apply for identity documents that can be used also by another individual. When a photo is brought in the photographer, the applicant, and others involved, all must trust that the photo has not been tampered with. In theory, biometric facial recognition algorithms should be able to determine altered photos from genuine ones, but, in practice, the algorithms are not perfect.

While the live enrolment of fingerprints is a common standard for identity documents, such as European passports, this is not the case for passport photos. The non- 
live photo enrolment procedure is vulnerable to 'morphing attacks' [1], in which a digitally altered photograph is enrolled in order to allow two or more persons of similar appearance to use the same passport to pass visual checks of their face (both automated and manual) at border crossing points. This would mean that a known criminal could travel with the passport of somebody else. More generally, if one does not make sure that the photo is an authentic representation of the person applying for the passport (and nobody else!), the photo will not provide the intended security. For instance, someone may be forced to apply for a passport with somebody else's photo, resulting in identitytheft where that somebody could travel in the name of the coerced. This could be useful for a criminal involved in e.g., illegal immigration or human trafficking. The adoption of a common standard for live enrolment of passport photos may eliminate, or at least greatly reduce, these threats.

As of 2016, only some European countries have switched to live enrolment (Figure 1).
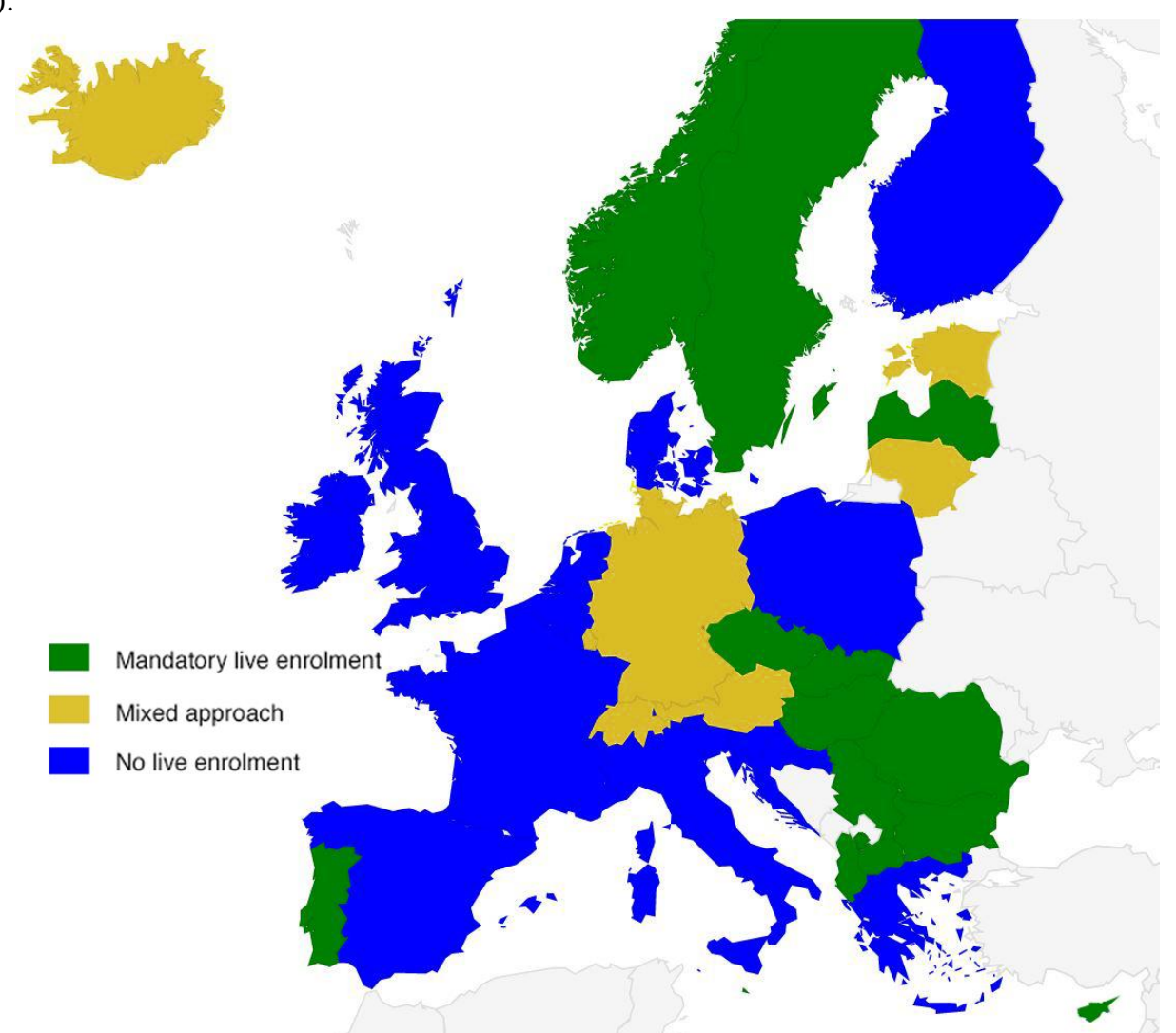

Fig. 1. State of the live photo enrolment in the Europe

Source: Authors on the basis of national experts participating in the "Sixty-sixth meeting of the Committee on a uniform format for visa" (16 March 2016, Brussels) and on national passport application websites. 
Some countries are applying a mixed approach where live enrolment is offered as an option, while most of the European countries are still applying traditional approach where applicants bring their photos with them. Primarily Northern and Southeastern European countries have made live photo enrolment mandatory. Meanwhile, a number of larger member states, such as France, Italy, Spain and the UK, have not yet launched live enrolment. In some cases, the existence of live enrolment varies at the intra-country level; for instance, it is available only in a third of Swiss cantons and various German municipalities.

The objective of the paper is to explain the main drivers and barriers behind switching to live enrolment. These are vital to understanding the problem at hand, its possible solutions, and to propose policy recommendations to other countries. It is important to note that there might not be one best model suitable for all countries as cultural and economic differences must be considered.

The research is carried out on the basis of four countries - Estonia, Kosovo (Kosovo is recognised as a country by most, but not all, EU member states), Norway and Sweden - in the issuance of passports. These countries represent a variety of practices. In Kosovo, Norway and Sweden live enrolment is mandatory. Estonia, on the other hand, represents a country where a mixed approach is in place. These countries represent an economically, socially, and culturally diverse group of countries, therefore they were selected as case study countries for this research.

As the first step, a review of existing academic and policy literature was conducted to search for influential factors that may affect the implementation of live enrolment. Since the specific topic is scarcely discussed in literature, the literature search was expanded to involve academic papers and policy reports on public sector innovation and technology acceptance. As a result, an inventory of potential barriers and drivers was compiled, covering technological issues, organizational, user aspects and other relevant factors.

For the second step, interviews were carried out with high-ranking persons at passport authorities of each country. Interviews were either conducted in person or by phone. The respondents - all government employees with a top-level passport process responsibility (currently or previously) - were selected to be knowledgeable and have good insight into the matter at hand.

The paper is structured as follows. In section two, a general overview of the live enrolment processes in Sweden, Norway, Kosovo and Estonia is provided. This is followed by literature review. In section 4 the formulation of several hypotheses about challenges and experiences of full live enrolment based on the theoretical studies and previous empirical work takes place. Section five discusses the findings, followed by the conclusions (section six). 


\section{Live Enrolment Processes in Sweden, Norway, Kosovo and Estonia}

In Sweden there is only one way of applying for a passport, and it is in person at a passport application office where a facial image is taken. This includes taking a digital photo and it is not possible for the applicant to receive a copy of the photo. The system was introduced in 2005.

Norway's process is highly similar to the Swedish one and was also introduced in 2005.

In Kosovo live enrolment is also in place, a digital photo has been taken close to the table of the clerk since 2008 .

Estonia, an example of a typical mixed enrolment process, accepts three different modes of the submission of passport photos since 2007:

- Live enrolment: Passport application with a photo taken in special photo booth, which links the digital photo with the personal identity code of the person. An important difference to e.g. the Swedish case is that the photo booths are self-service rather than operated by application office staff. The differences may have implications for both quality and security.

- Traditional application for passport on a paper along with printed photo, submitted in person in Estonian Police and Border Guard Board or sent in by postal mail;

- Electronic passport application (filled in PDF form) together with photo in JPG format in the same electronically signed document (BDOC).

\section{$3 \quad$ Literature Review}

Studies on ICT-driven innovation in the public sector frequently emphasize the organizational, administrative and political context as a source of innovation drivers and barriers. Since the application of the live enrolment requires changes in organizational workflows and coordination between different organizations, it faces a number of organizational barriers. Such barriers involve, for example, existing governmental silos and lack of communication, the complexity of organizational change, and concerns about high implementation costs.

Studies have found that the innovation capacity of public sector organizations depends on a number of organizational factors, such as organizational structures, intraand inter-organizational collaboration and coordination, organizational culture, leadership styles [2-3], resources [4], qualified personnel [5], etc. Public sector organizations are also strongly affected by the legal culture and administrative traditions of the state [4]. Moreover, the inherent complexity of public sector organizations and their accountability to a multitude of stakeholders make the implementation of organizational changes much more difficult in the public sector than it is in the private sector [6].

Although the public sector context generates more barriers than drivers to innovation, some drivers still exist. Firstly, drivers may be generated by external triggers, such as competitive pressure by other organizations, countries or international bodies [4], 
legal obligation, political priority and public demand [2]. At the organizational level, innovation can be driven by participation in cross-organizational and cross-border knowledge transfer networks [7], and strong leadership by administrative and political managers [4].

The regulatory and legal context is perceived to be important in several respects. On the one hand, existing regulations often stifle innovation; on the other, regulations can also promote innovation, for example by imposing a legal obligation to implement certain solutions [2].

Another potentially important factor is demand by citizens and businesses and can act as an influential factor for live enrolment. In order to understand what factors affect the demand and acceptance of live enrolment, research on the acceptance and use of technology can provide valuable insights. The general point of departure of such literature is the understanding that there are a number of factors that influence the user as to whether or not to adopt a novel technology. One popular approach for mapping those factors is the technology acceptance model (TAM), which argues that acceptance is determined by the perceived usefulness and ease of use of a technology [8]. TAM's derivative, the Unified Theory of Acceptance and Use of Technology (UTAUT) is more elaborate and incorporates additional factors, explaining how a decision is formed about the use of an information system. The theory builds on four key constructs: 1) performance expectancy, 2) effort expectancy, 3) social influence, and 4) facilitating conditions [9-10].

\section{Hypotheses}

On the basis of the conducted theoretical research and previous empirical work, we have formulated several specific hypotheses about barriers and drivers of full live enrolment. This includes aspects pertaining to both the users - individuals expected to use the live enrolment service - as well as the suppliers of said public service.

Our key hypotheses are related to the expectations of the government when introducing live enrolment. We expect security related benefits, such as making sure the photo comes from the person who is applying for a passport and is not altered in any way as well as other kinds of protection from document forgery. In addition, central arguments around introducing live enrolment relate to the accuracy and reliability of the identification of persons and protection from identity theft. We also expect to see more general public security policy objectives, such as the fight against illegal immigration and human trafficking, as well as the fight against terrorism and serious crime. [11], [12]

We expect the general key barriers and drivers of public sector innovation to be the same for the specific case of live enrolment, with the importance of continuous political support to the process, financial resources, commitment and leadership of administrative and technical managers ("championing of the project"), ICT awareness and capabilities of the stakeholders (systems, skills, tools and methods) [2], [3], [13].

Existence of a suitable live enrolment technology on the local market, or suppliers who can provide it, might also be important as well as related business models. The 
cost of implementing live enrolment may also be mitigated by similar existing systems and solutions. If live enrolment for ID cards is already in place, the step towards live enrolment for passports should be considerably easier. Extensive population censuses (registries) can further help as there will be more information in the system already. Conversely, going for live enrolment could also be made easier if other changes were anyway needed to the passport application process, such as introducing fingerprint scanners.

Any security measure must be cost-efficient and in some cases it may be that population density (e.g. in relation to the number of application offices) is too low to offset the cost of live enrolment. On the other hand, live enrolment could be a more costeffective security measure than e.g. ones based on complex certificate distribution, or instead less cost-effective than putting a limit to the amount of new passports a citizen is allowed in a set period of time.

As public sector innovation is risky, we expect to see evidence of resistance from some stakeholders (e.g., agencies reluctant to take on additional tasks). This may be particularly likely in case procedures were recently changed. Related to this is the use of formalized methodological approaches to implementing the change (a clear definition of work rules and methods, training, etc.) in order to mitigate the risks. Since general experience in public sector innovations is that only limited attention is paid to precise systematic gathering and use of measurement and data, we do not expect to see very clear measurable target indicators set in relation to live enrolment.

There are also certain probable expectations from the public which can influence live enrolment uptake [14]. Convenience of the collection of photos (e.g. easier to use photo booth than make the extra effort to a professional photographer) and image satisfaction are likely important factors. Furthermore, the spread of digital cameras could have a two-sided effect: on the one hand, encouraging uptake of related technologies such as live enrolment, while on the other hand, resistance from professional photographers due to an increasingly smaller market may appear. We also expect to see some problems due to the innovative nature of technology. Societal groups such as those less familiar with technology and digital photography may be more risk-averse and resistant to live enrolment, preferring the traditional method; age and profession distribution of a region or country may be underlying factors here. Pre-existing widespread use of privacy-sensitive technology, like biometrics and databases, may make live enrolment easier to accept. For instance, fingerprint usage has previously had bad connotations due to the connection with criminal records, but this has been alleviated by the rising use of fingerprint logins on smartphones.

Finally, since the main purpose of passports is to travel to other jurisdictions, a country must consider not only its own perceptions but also external pressure. For instance, countries must take into account demands from the EU and International Civil Aviation Organization (ICAO) as well as particular countries like the US requiring certain procedures to ensure visa-free entry.

These specific hypotheses were the main topics focused of the interviews and other data collection, and in the following section key findings are discussed. 


\section{Discussion}

If current facial recognition algorithms are unable to fully deal with digital image alterations, and live enrolment- where photos are taken in a controlled environment in the application office - provides a way of alleviating this risk, then the question becomes why are all countries not already using it. Our research shows that some countries have switched to live enrolment successfully, while many still use the traditional method of having citizens bring photos when applying for a passport. Also, many countries allow both methods.

Building on the theories of public sector innovation and acceptance and use of technology, the authors have identified a number of factors influencing live enrolment decisions and implementations. These factors may be split in those behind the decision to switch to live enrolment and the factors impacting the ease of implementation.

\subsection{Making the Decision to Go Live}

A main driver for live enrolment is increased security, hindering false images in passports. Furthermore, live enrolment is convenient for citizens as they do not need to first acquire photos before visiting the application office. Also, formatting issues are unlikely to occur if all photos are taken by the same organisation. However, all security measures must be weighed against the different associated potential costs. For instance, the passport authority will need to administer photos in a different way, while portrait photographers lose a fairly substantial part of their income. Photos not taken by experienced photographers may also lead to less flattering portraits, which may be associated with a decrease in satisfaction among citizens. Some photographers have indicated that they believe they could provide as secure an image process as the government officials and this may ultimately be a question of trust.

There may also be old connotations of feeling like a criminal attached to biometrics being captured by the authorities and care must be taken to give applicants sufficient privacy, especially for those with certain religious beliefs or disabilities. Indeed, the passport authorities in Sweden and Norway are both trying to be service minded and create good will.

Another factor affecting the decision of switching to live enrolment is the external global pressure from organisations like the EU and ICAO, as well as influential countries like the USA who require certain procedures for visa free entry. In general, however, EU member states seem to be doing very well with passport security in an international context. There may be pressure coming from other countries having implemented live enrolment or incentives coming from other parts of one's own government who use similar technology. On the other hand, traditions may be strong and if a country recently changed its application procedures it will likely be more reluctant to do so again.

It is also interesting to note that the motivation for going live has been rather different in our case study countries. Estonian prioritised the quality of photos, as the photos were previously not always of sufficient quality, while security considerations, such as avoiding photo morphing were not of concern. Contrastingly, for Kosovo, Sweden and 
Norway security considerations were the highest. For Estonia, user convenience has been very important and is behind the decision of keeping alternative application processes open for the citizens.

Another finding is that in the absence of legacy systems (like in Kosovo), more profound decisions are generally reached (and implemented) in an easier fashion. Also, it must be reiterated that decisions are easier to reach if there are other organisations that have introduced live enrolment for related services - it is possible to learn from their experience and somehow pressure to innovate is created from the public side if they have positive experience with live enrolment in other context.

In general, still, decisions to employ live enrolment seems to be influenced equally both by the context external to public sector organizations as well as by organisational level factors. External pressures alone (such as security related considerations by politicians) cannot explain the decisions to switch, as more countries would have switched to live enrolment by now. Suitable organizational level context (including supportive organisational culture, allocation of resources), coupled with individual level drivers of key persons (such as job-related knowledge and skills, willingness to exploit risky avenues) behind the live enrolment introduction, were also needed.

\subsection{Implementing Live Enrolment}

Even if the decision is made to introduce live enrolment, proper risk management is needed to account for any and all setbacks encountered during implementation. One possible risk is that appropriate technology is missing or that vendors do not have suitable offerings. There are not many vendors available and, as such, there is low competition that may have led to higher prices and doubtful quality. There are ideas of certifying vendors to address this. One of the typical problems related to public sector innovation is related to the lack of suitable technology in market. Thus, there is need to develop related technologies further until they can be applied. This also calls for the implementation of rather unpopular public procurement mechanism, so-called public procurement of innovation. In the case of live enrolment suitable solutions did exist in the global market. However, it should be noted that any vendor winning a particular procurement process will effectively have a monopoly for live enrolment in the applicable region and time of that procurement. As such, there is considerably more at stake than in the case of non-live enrolment where there can be many simultaneous vendors sharing the market. Thus, care must be taken in order not to let corruption distort the procurement process.

Furthermore, staff must be trained. In an unstable political situation, a decision may soon be reversed and idea championing and motivation could be limited. Overall, a culture rife with innovation will be more accustomed and prepared for changes and therefore more likely to succeed with a change in enrolment processes. Of special concern is when passport applications are done for extraordinary circumstances, such as when conducted outside of one's country, when applying for temporary/emergency passports, or when applying for visas. Indeed, citizens seem to highly value the speed of the application process, with examples of citizens making sure they get passports with the old ten year validity before a switch to a mere five year validity. In general, 
citizens seem more concerned about the use of fingerprints and biometric data in databases than about live enrolment. Passport officials have suggested changing the discourse and focusing on the improved security measures to stop identity theft.

Our research confirms that one of the typical weaknesses associated with insufficient attention to the use of impact assessments and evaluations is also true regarding live enrolment. None of the countries set target indicators nor was their achievement monitored. Neither have we encountered (public) impact assessments of the benefits of live enrolment (that in turn might slow down other countries to switch as the benefits are debatable).

Our research of technology acceptance aspects did not reveal problems. The overall societal context was supportive as people had already experience with digital photography and automated service machines generally.

\subsection{A Multi-faceted Situation}

The interviews revealed varying experiences, obstacles, cultural differences and tradeoffs. Only in Sweden was the voice of the photographers an issue. Indeed, Estonia may have found a move towards live enrolment easy since they also kept the traditional application method. However, Norway also went full live and in fact had a lot of political support and funding, although the support and funding varied over time. Kosovo, being a rather young country, seems to have succeeded with implementing live enrolment thanks to no pre-existing alternatives. While there were some discussions among photographers, their voices were not very strong. Furthermore, the issue was not particularly political, in part thanks to a small government. Also, since Kosovo's efforts started already in 2000 , there was no issue with the advent of digital cameras further affecting photographers.

The other parts of the identification chain were also important influencers. The existence and levels of birth registries vary widely in the EU, as do the use of automatic border gates, mobile application kiosks and restrictions on citizens' right to a passport. The use and sophistication of other forms of ID may also play a role. For instance, the Swedish transport authority previously tried but failed to implement live enrolment for driver's licenses. Now they plan to revisit this with the experiences of the passport authority.

\section{Conclusions}

Using live enrolment for passports, where photos are taken in the application office, is a topical issue since it can limit the possibility of malicious digital image alterations [15]. However, live enrolment has both its advantages and disadvantages and a tradeoff is expected. Live enrolment may make passport applications a one-stop process, ensure consistent formatting and increase security. There may also be spill-over effects. For example, Estonia first introduced live enrolment for driver's licences, and this ex- 
perience simplified introduction of the live enrolment for passports. Sweden is following a reversed path from live passport enrolment making the similar move for licences simpler. External pressure can also be international e.g. from the ICAO or EU.

However, live enrolment is a break with tradition and depends on overhauls in the public sector where measurable targets and risk management is typically wanting and the passport authority may be reluctant to take on new tasks. The public may hesitate to trust the government further with their private data and find that their portraits are now less flattering. Furthermore, live enrolment can make it more difficult for expats, who need to apply for a passport from abroad. Furthermore, a lack of competition among live enrolment equipment vendors may lead to high prices for low quality, which both Sweden and Norway have found. Portrait photographers meanwhile suffer loss of income.

To minimise the trade-off, authorities have tried becoming more service-minded, creating good will, not least in relation to the speed of the application process, which is highly important to applicants. Portrait photographers, on the other hand, have suggested that they be certified, in a similar vein to what is done in other areas where the private sector provide a service for the public good, thereby ensuring adequate levels of security. However, at least so far their suggestions have not become a reality. In the case study of four countries, only in Sweden was the voice of the photographers at all an issue and even there the authorities chose to prioritise security and live enrolment.

The decision to introduce live-enrolment and the state of live enrolment in Europe are both multi-faceted. Experiences, drivers and obstacles vary between countries and sometimes there are even more regional structures where one part of a country differs from another. Furthermore, it is not a binary decision: there are different versions of live enrolment implementations and also mix enrolment where live enrolment is offered but not mandatory, such as in Estonia. What is more, when planning the introduction of live enrolment, Estonia prioritised quality of photos and user-friendliness while Sweden focused on security. Implementation wise, Kosovo benefitted from a small government and no pre-existing alternatives, while also pre-empting the digital camera era which later put more pressure on photographers. Norway had substantial political support and funding, although the backing varied over time, while Estonia utilised Schengen funds.

As such, the decision to introduce live enrolment and successfully implementing it, is dependent on a vast number of cultural and political factors. Straightforward sociocultural models are unable to fully explain the current situation and the overall state of a country's passport maturity with its automatic border gates, mobile application kiosks, restrictions on passport renewals as well as the proliferation of birth registries and the security of breeder documents.

Several limitations remain, however. First, the attitudes of photographers (as important stakeholder group) could be studied further. They might be hard to reach, though, as those who suffered economically due to live enrolment could no longer be in business. Also, empirical data could be collected from the countries that do not practice live enrolment now to deepen current results. 


\section{Acknowledgments}

This work was supported by the European Commission through the project "Fast and trustworthy Identity Delivery and check with ePassports leveraging Traveler privacy" (FIDELITY, grant 284862) funded from FP7 and a grant "Public sector innovation: The case of modern identity management technologies" (PUT773) from Estonian Science Foundation.

\section{References}

1. Ferrara, M., Franco, A., Maltoni, D. The Magic Passport. IEEE International Joint Conference on Biometrics (IJCB), 1-7 (2014).

2. De Vries, H., Bekkers, V., Tummers, L. Innovation in the Public Sector: A Systematic Review and Future Research Agenda. Public Administration 94(1), 146-166 (2016).

3. European Commission. Powering European Public Sector Innovation: Towards A New Architecture. Report of the Expert Group on Public Sector Innovation (2013).

4. Bekkers, V., Tummers, L., Voorberg, W. From public innovation to social innovation in the public sector: A literature review of relevant drivers and barriers. Rotterdam: Erasmus University Rotterdam (2013).

5. Alshehri, M., Drew, S.J. E-government principles: implementation, advantages and challenges. International Journal of Electronic Business 9, 255-270 (2011).

6. Rashman, L., Withers, E., Rashman, J.H. Organizational learning and knowledge in public service organizations: A systematic review of the literature. International Journal of Management Reviews 11(4), 463-494 (2009).

7. Albury, D. Fostering Innovation in Public Services. Public Money \& Management 25, 5156 (2005).

8. Davis, F.D. Perceived usefulness, perceived ease of use, and user acceptance of information technology. MIS Quarterly 13(3), 319-340 (1989).

9. Venkatesh, V. Determinants of Perceived Ease of Use: Integrating Control, Intrinsic Motivation, and Emotion into the Technology Acceptance Model. Information Systems Research 11(4), 342- 365 (2000).

10. Venkatesh, V., Thong, J.Y.L., Xu, X. Consumer acceptance and use of information technology: extending the unified theory of acceptance and use of technology. MIS Quarterly 36(1) 157-178 (2012).

11. Hoepman, J.-H. , Hubbers, E., Jacobs, B. ., Oostdijk, M., Schreur, R.W. Crossing Borders: Security and Privacy Issues of the European e-Passport, 1st Int. Workshop on Security, Kyoto, Japan, October 23-24. http://arxiv.org/abs/0801.3930 (2006).

12. Tiits, M., Kalvet, T., Laas-Mikko, K. Social Acceptance of ePassports. Lecture Notes in Informatics P-230, 15-26. (2014).

13. Kalvet, T. Innovation: a factor explaining e-government success in Estonia. Electronic Government, an International Journal 9, 142-157 (2012).

14. Kalvet, T., Tiits, M., Laas-Mikko, K. Public Acceptance of Advanced Identity Documents. In Proceedings of the 11th International Conference on Theory and Practice of Electronic Governance (ICEGOV'18), Galway, Ireland, April 2018 (2018).

15. Behrensen, M., Kalvet, T., Kindt, E., Tiits, M., Palm, E. Harmonization of ePassport and Border Checks in the European Union: Ethical, Social, and Legal Aspects. A summary of the practical implications of the legal, ethical, and social analysis of the FIDELITY project (2016). 